\title{
Ibrahima Diop Gaye (dir), Mobilité pastorale et développement au Sahel
}

Irène Mestre

\section{OpenEdition}

Journals

Édition électronique

URL : http://journals.openedition.org/geocarrefour/10471

ISSN : 1960-601X

Éditeur

Association des amis de la Revue de géographie de Lyon

Ce document vous est offert par BU de l'Université Jean Moulin Lyon 3

JEAN MOULIN

\section{Référence électronique}

Irène Mestre, « Ibrahima Diop Gaye (dir), Mobilité pastorale et développement au Sahel », Géocarrefour [En ligne], Comptes rendus inédits, mis en ligne le 29 mai 2018, consulté le 29 octobre 2018. URL http://journals.openedition.org/geocarrefour/10471

Ce document a été généré automatiquement le 29 octobre 2018

(c) Géocarrefour 


\section{Ibrahima Diop Gaye (dir), Mobilité pastorale et développement au Sahel}

Irène Mestre

\section{RÉFÉRENCE}

GAYE I. D. (dir), 2017, Mobilité pastorale et développement au Sahel, Dakar, L'Harmattan

Sénégal, $287 \mathrm{p}$.

1 Cet ouvrage présente un bilan du travail du groupe d'intérêt scientifique Pôle Pastoralisme et Zones Sèches (PPZS) né du partenariat entre des institutions sénégalaises et internationales. Le PPZS vise à explorer les questions liées au pastoralisme et au sylvopastoralisme dans la région du Sahel pour faire avancer la recherche et contribuer à l'enseignement. Il s'appuie pour cela sur une équipe de recherche pluridisciplinaire répartie entre la France, le Sénégal et le Kenya. Les questionnements scientifiques soulevés sont ancrés dans les problématiques de développement des zones rurales. La préface de Madame Aminata Mbengue Ndiaye, Ministre de l'élevage et des productions animales du Sénégal souligne l'importance de l'étude de la mobilité pastorale dans un contexte où le pastoralisme est souvent perçu comme une activité archaïque et nocive pour les écosystèmes. Cet ouvrage explore, à travers des prismes, des échelles et des disciplines différents, la mobilité pastorale dans la région sahélienne.

2 S'appuyant sur des études de cas et des données de terrain, l'ouvrage est divisé en deux parties et onze chapitres. Dans la première partie est abordée la notion de mobilité à travers les questions de développement qu'elle soulève. En effet, les systèmes pastoraux sont à la fois un mode de production, une culture et un système de connaissance, du fait même de leur structuration. Cette mobilité répond à des schémas variés qui dépendent d'un grand nombre d'éléments. Elle interroge en effet des domaines aussi variés que la protection des écosystèmes et, en particulier, des forêts au Sénégal (chapitre 2, rédigé par 
Oumar Ndao Gning, Oumar Sarr, Paul Ndiaye et Léonard-Elie Akpo), la gouvernance à l'échelle nationale et dans les communes septentrionales (chapitre 3 rédigé par Ibrahima Diop Gaye et Alioune Bâ et chapitre 4 rédigé par Oumar Sy) et les stratégies d'individuation de la mobilité à partir de cas d'étude dans le Ferlo (chapitre 5 rédigé par Astou Diao Camara et Véronique Ancey). A travers cet ensemble de cas, se dessine la richesse des facteurs qui façonnent la mobilité et au-delà les dynamiques pastorales. Celle-ci apparait comme le résultat d'une stratégie prenant en compte un faisceau d'éléments dans lequel les besoins du troupeau jouent un rôle clé sans pour autant être singulier. La deuxième partie s'appuie sur ce constat pour l'analyse des évolutions des formes et de la nature même des mobilités pastorales en combinant différentes disciplines. La mobilité pastorale a toujours été très liée aux réseaux de commercialisation, soit en adaptant les déplacements, par exemple entre le Sahel et les pays côtiers (chapitre 7 rédigé par Christian Corniaux, Brigitte Thébaud et Denis Gautier), soit en favorisant des circuits compatibles comme présenté dans l'étude du cas d'une laiterie dans le Ferlo (chapitre 11 rédigé par Abdrahmane Wane, Alioune Ndiaye, Ibra Touré et Ibrahima Kane). Il en va de même pour la coordination des mesures de soutien aux services vétérinaires dans le cadre de l'élevage mobile (chapitre8 rédigé par $\mathrm{A}$. $\mathrm{T}$. Diop, O. T. Diaw et A. G. Fall). A travers tout le Sénégal, le mitage des espaces ruraux et la faible prise en compte des parcours, sont un frein important au pastoralisme. Malgré un discours politique qui promeut la mise en place de plans concertés, celle-ci est souvent faible (chapitre 6 rédigé par Amadou Tamsir Diop). Si la mobilité est souvent étudiée à l'échelle régionale ou nationale, l'étude des dynamiques qui sous-tendent les déplacements des troupeaux permet de mieux comprendre les interactions troupeauenvironnement et donc de mieux anticiper les changements induits par des modifications des écosystèmes (chapitre 10 rédigé par Mamadou Sané, Jonathan Vayssières, Myriam Grillot, Alassane Bah et Alexandre Ickowicz). Face au constat de l'échec des outils classiques de l'aménagement du territoire, Alassane Bah, Christine Fourage et Ibrahima Diop Gaye présentent dans le chapitre 9 une étude de cas dans la région du lac de Guiers sur une expérimentation de modélisation s'appuyant sur le concept de système socioécologique. L'objectif poursuivi est de représenter la complexité des systèmes pastoraux pour développer un outil collaboratif - entre chercheurs, usagers et gestionnaires.

3 De nombreuses questions sont abordées de manière transversale par différents chapitres. Dans la région sahélienne, le pastoralisme doit s'adapter à des régimes de frontières de plus en plus stricts et à la faible coordination des politiques liées aux déplacements, notamment concernant la santé animale entre les pays. Le renforcement du contrôle des Etats centraux sur les espaces de marges met en péril les pratiques pastorales transfrontalières sans pour autant offrir d'alternatives.

4 La pression foncière est un enjeu crucial abordé à plusieurs reprises dans l'ouvrage. Les différents usages du territoire tendent à s'exclure mutuellement. Ainsi, l'urbanisation et le développement provoquent la rupture des systèmes de ressources nécessaires au pastoralisme. Dans tous les cas étudiés, les auteurs s'interrogent sur les articulations avec les politiques publiques et en particulier sur l'intégration des activités et des populations pastorales dans l'élaboration et la mise en place des politiques. Les chapitres 9 et 10 offrent des perspectives intéressantes sur les outils pour la réflexion et pour l'action collaborative en décrivant des outils de modélisation des systèmes à l'échelle de l'exploitation et du système pastoral. 
5 De manière générale, cet ouvrage dépeint la richesse des interactions entre les systèmes pastoraux et non-pastoraux. Il est important de noter que les auteurs de cèdent pas à la généralisation et n'hésitent pas à mettre en avant des dynamiques contradictoires, entre certains systèmes pastoraux dont l'intégration s'intensifie du fait de stratégies souples et d'un environnement favorable, et des populations et des espaces pastoraux qui se marginalisent. De la même manière, la variété des cas d'étude permet de dépasser les orthodoxies des études des systèmes pastoraux.

6 Cet ouvrage, présente de façon à la fois documentée et ancrée sur le terrain les caractéristiques et les dynamiques du pastoralisme au Sahel. La combinaison des approches disciplinaires offre des pistes pour l'étude des facteurs et des effets des évolutions de la mobilité pastorale. A travers la grande variété de cas d'étude, de méthodes et des zones d'études, se dresse le constat de l'étonnante capacité des systèmes pastoraux à s'adapter et se transformer, tant aux changements extérieurs qu'aux évolutions internes auxquels ils font face. Nous ne pouvons que souhaiter que l'équipe du PPZS poursuive ses recherches et produise des ouvrages explorant de manière transversale et approfondie les thématiques abordées ici.

\section{AUTEURS}

\section{IRÈNE MESTRE}

CNRS UMR 5600 Environnement, ville, société irene_mestre@hotmail.com 